$\underline{\xi}=\mathbf{a}$

\title{
Laser orbital perturbations in Hamiltonian framework
}

\author{
El-Saftawy M. I. ${ }^{1,2}$ *, Nabawia S. Khalifa ${ }^{2,3}$ \\ ${ }^{1}$ King Abdul-Aziz University, Faculty of Science, Department of Astronomical Science, Jeddah, K.S.A \\ ${ }^{2}$ National Research Institute of Astronomy and Geophysics, Helwan, Cairo, Egypt \\ ${ }^{3}$ Hail University, Department of Mathematics, Hail, KSA \\ *Corresponding author E-mail: malsaftawy@kau.edu.sa
}

\begin{abstract}
The effect of laser photon pressure on the spacecraft's orbit is modeled. The force model developed taking into consideration atmospheric beam attenuation. However, adaptive optics assumed fixed on the laser system in order to eliminate the effect of atmospheric turbulence. The force of a single laser pulse proved as a conservative force. Consequently, its potential obtained using Legender polynomial. Assuming a spherical Earth, the Hamiltonian of the problem developed in terms of Dalaunay elements up to the first zonal harmonic. Using different ground-based laser systems, the model applied to the satellite Ajisai of NORAD ID 16908 and the LEO debris ASTRO-F DEB of NORD ID 29054. The numerical results emphasized that laser pressure has an effect on the orbit and it is well agreed with the results of the Newtonian treatment of the problem.
\end{abstract}

Keywords: Canonical Formulation; Laser Radiation Pressure and Orbital Perturbations

\section{Introduction}

Recently, a rapid development and utilization of laser applications were achieved in all scientific branches particularly in space (e.g. satellite laser ranging, laser space debris removal, laser propulsion, laser broom... etc). Most of the authors depended in their studies on laser thermal effects, which result in ablation and vaporization of the projected surface. These effects were applied for space debris removal or reentry [1]. In 2004, El-Saftawy M. I., et al. stared a new study of laser space applications which based on the laser dynamical effect on the satellite orbit. They constructed a simple force model in absence of atmospheric effects on laser beam propagation [2, 3]. In 2008, the force model developed to include the effect of the Earth's atmosphere and a comprehensive study of laser effect on the orbital elements in the Newtonian framework was performed [4]. The main conclusion of those studies is laser force has a reasonable effect on satellite orbital elements [5]. 2011, James Mason et al. investigated the feasibility of using a medium power ground-based laser for space debris removal. They concluded that medium power $(5-10 \mathrm{~kW})$ groundbased laser can provide sufficient photon pressure which can substantially perturb low-Earth Sun-synchronous debris fragments in few days [6].

The main issue of that current paper is to study laser pressure effect on satellite orbital element in the Hamiltonian framework.

\section{Laser force as conservative force}

For flat non-perfectly reflecting surfaces, the laser force affecting on the satellite surface is given by [9]:

$$
\bar{F}=\frac{S(\rho) A}{C} \Psi \operatorname{Cos} \Theta \hat{\rho}
$$

Where $\Theta$ is the cone angle, $\hat{\rho}$ is the radiation incident direction, $A$ is the projected area, $\mathrm{C}$ is the speed of light, $\rho$ is the satellite range, $S(\rho)$ is the laser beam intensity and $\Psi$ is a function of satellite thermo-optical properties which is given by:

$$
\begin{aligned}
\Psi & =\left\{4 \rho^{\prime} \beta \cos ^{4} \eta+2\left(1+\rho^{\prime} \beta\right)\left[\begin{array}{l}
\boldsymbol{B}_{f} \rho^{\prime}(1-\beta) \\
\left.+\alpha^{\prime}\left(\frac{\varepsilon_{f} \boldsymbol{B}_{f}-\varepsilon_{b} \boldsymbol{B}_{b}}{\varepsilon_{f}+\varepsilon_{b}}\right)\right] \cos ^{3} \eta+
\end{array}\right.\right. \\
& +\left[\left(1+\rho^{\prime} \beta\right)^{2}+\left(\begin{array}{l}
\boldsymbol{B}_{f} \rho^{\prime}(1-\beta) \\
\left.\left.\left.+\alpha^{\prime}\left(\frac{\varepsilon_{f} \boldsymbol{B}_{f}-\varepsilon_{b} \boldsymbol{B}_{b}}{\varepsilon_{f}+\varepsilon_{b}}\right)\right)^{2}\right] \cos ^{2} \eta\right]
\end{array}\right]^{\frac{1}{2}}\right.
\end{aligned}
$$

Where $\eta$ is the radiation incident angle w.r.t. the surface normal, $\beta$ is the surface specularity, $\rho^{\prime}$ is the surface reflectivity, $B_{f}$ and $B_{b}$ are the non-Lambartian coefficient of front and back surfaces respectively, $\alpha^{\prime}$ is the absorption coefficient, and $\varepsilon_{\mathrm{f}}$ and $\varepsilon_{\mathrm{b}}$ are front and back surface emissivity respectively. The value of $\Psi$ is ranged from 1 to 2 ; where $\Psi=0$ for transparent surfaces, $\Psi=2$ for perfectly reflecting surfaces and $\Psi=1$ for opaque surfaces.

For standard atmospheric conditions, only linear mechanism of laser atmospheric interactions is considered. Atmospheric turbulences are countered by using the adaptive optics and technical capabilities of the laser system. Based on the previous postulates, the laser intensity delivered to the satellite surface is determined using an analytical model in which laser intensity is proportional directly to the laser power and inversely to satellite altitude and laser divergence. This laser intensity is given by [7], [5]:

$$
S(\rho)=\frac{P_{o}}{\pi} \frac{K}{\rho^{2}}
$$

Where $\mathrm{K}$ represents the effect of the Earth's atmosphere on the laser beam propagation and it can be given by: 


$$
\mathrm{K}=\frac{1}{\theta^{2}} \exp \left[-\left(\frac{\sigma_{\mathrm{scat}}^{\mathrm{mol}}(0) \mathrm{h}}{\sin \phi}\left(1-\exp -\left(\frac{\mathrm{w} \sin \phi}{\mathrm{h}}\right)+\sigma_{\text {scat }}^{\mathrm{aer}}\right)\right.\right.
$$

Where $P_{0}$ is the laser initial power, $\theta$ is the beam divergence, $\phi$ is the elevation angle, $\sigma_{\text {scat }}^{\mathrm{mol}}(0)$ is the molecule scattering coefficient at sea level, $\sigma_{\text {scat }}^{\text {aer }}$ is the aerosols scattering coefficient and $h$ is the sea level altitude which is considered to be $7 \mathrm{~km}$. Since the most effective region on the beam propagation is considered to be the first $50 \mathrm{~km}$ of the Earth's atmosphere, $w$ is chosen to be $50 \mathrm{~km}$ [8]. For laser beam of wavelength $(\lambda=532 \mathrm{~nm})$, the molecular scattering coefficient at the sea level $\sigma_{\text {scat }}^{\text {mol }}(0)=1.7 \times 10^{-5} \mathrm{~m}^{-1}$ .while for clear weather condition, the aerosols scattering coefficient $\sigma_{\text {scat }}^{\text {aer }}=0.0001 \mathrm{~m}^{-1}$ [9]. Equation (1) can be rewritten as:

$\overline{\mathrm{F}}=\varepsilon \Psi_{1} \frac{\widehat{\rho}}{\rho^{3}}$

Where $\Psi_{1}=\mathrm{KA} \Psi \cos \Theta$ and $\varepsilon=\frac{\mathrm{P}_{\mathrm{o}}}{\pi \mathrm{c}}$.

Assuming that $\hat{\mathrm{e}}_{\mathrm{x}}, \hat{\mathrm{e}}_{\mathrm{y}}$ and $\hat{\mathrm{e}}_{\mathrm{z}}$ are three unit vectors of a geocentric coordinate system xyz. In which $\hat{e}_{\mathrm{x}}$ is directed parallel to the Earth equatorial plane, $\hat{\mathrm{e}}_{\mathrm{y}}$ is directed in the plane that contains the meridian of the sub-satellite point and $\hat{\mathrm{e}}_{\mathrm{z}}$ directed normal to the equatorial plane, as described by [10]. For a single laser shot, the Curl of laser force is given by:

$$
\begin{aligned}
\operatorname{Curl} \overline{\mathrm{F}} & =\left|\begin{array}{ccc}
\hat{\mathrm{e}}_{x} & \hat{\mathrm{e}}_{y} & \hat{\mathrm{e}}_{z} \\
\frac{\partial}{\partial x} & \frac{\partial}{\partial y} & \frac{\partial}{\partial z} \\
F_{x} & F_{y} & F_{z}
\end{array}\right| \\
& =\left(\frac{\partial F_{z}}{\partial y}-\frac{\partial F_{y}}{\partial z}\right) \hat{\mathrm{e}}_{\mathrm{x}}+\left(\frac{\partial F_{z}}{\partial x}-\frac{\partial F_{x}}{\partial z}\right) \hat{\mathrm{e}}_{y} \\
& +\left(\frac{\partial F_{y}}{\partial x}-\frac{\partial F_{x}}{\partial y}\right) \hat{\mathrm{e}}_{z} \\
& =-\frac{3 \Psi_{t}}{\rho^{5}}\left[\begin{array}{l}
\left.\left(\rho_{y} \rho_{z}-\rho_{z} \rho_{y}\right) \hat{\mathrm{e}}_{x}+\left(\rho_{x} \rho_{z}-\rho_{z} \rho_{x}\right) \hat{\mathrm{e}}_{y}\right]=\overline{0} \\
+\left(\rho_{y} \rho_{x}-\rho_{x} \rho_{y}\right) \hat{\mathrm{e}}_{z}
\end{array}\right]
\end{aligned}
$$

Since, the laser force $\overline{\mathrm{F}}$ meets the condition, $\operatorname{Curl} \overline{\mathrm{F}}=\overline{0}$, so it can be considered as a conservative force.

\section{The laser disturbing potential}

Since laser force is conservative, it is possible to assign a numerical value for the potential at any point. Assuming that the potential function is $\mathrm{u}(\mathrm{x}, \mathrm{y}, \mathrm{z})$ so that

$$
\overline{\mathrm{F}}=-\nabla \mathrm{u}
$$

\section{Consequently:}

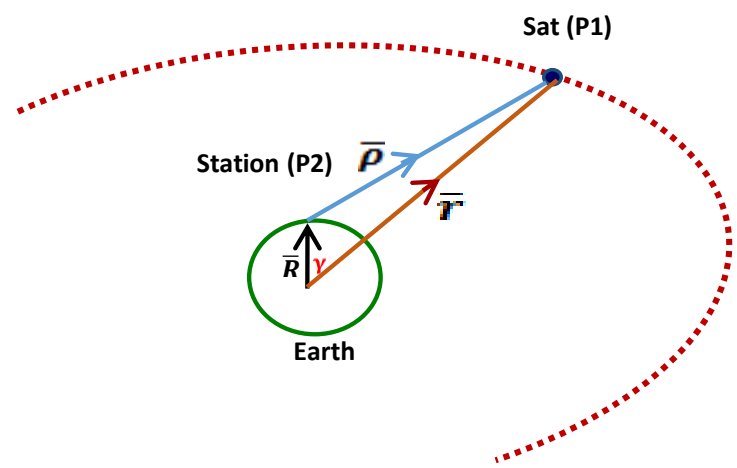

Fig. 1: Laser Intensity Delivered to the Satellite Surface.

$$
\mathrm{u}(\mathrm{r})=-\int \overline{\mathrm{F}} \cdot \mathrm{d} \overline{\mathrm{r}}=\frac{3 \varepsilon \Psi_{1}}{\rho}
$$

Using fig. 1, the range vector is given by:
$\bar{\rho}=\overline{\mathrm{r}}-\overline{\mathrm{R}}$

With magnitude

$\rho=\sqrt{\bar{\rho} \cdot \bar{\rho}}=\sqrt{r^{2}+R^{2}-2 r R \operatorname{Cos} \gamma}=r \sqrt{1+\left(\frac{R}{r}\right)^{2}-2 \frac{R}{r} \operatorname{Cos} \gamma}$

Where $\bar{R}$ is the geocentric station coordinate vector and $\bar{r}$ is the satellite position vector. Let $\chi=\frac{R}{r}$, then:

$\mathrm{u}(\mathrm{r})=\frac{3 \varepsilon \Psi_{1}}{\mathrm{r}}\left(1-2 \chi \cos \gamma+\chi^{2}\right)^{-\frac{1}{2}}$

Since $R<\mathrm{r}$, then $\chi<1$ and the laser potential can be expanded in a convergent power series of the form:

$\mathrm{u}(\mathrm{r})=\frac{3 \varepsilon \Psi_{1}}{\mathrm{r}} \sum_{\mathrm{n}=0}^{\infty} \chi^{\mathrm{n}} \mathrm{P}_{\mathrm{n}}(\cos \gamma$

Where $P_{n}(x)$ are the conventional Legendre polynomials defined as:

$$
P_{n}(x)=\frac{1}{2^{n} n !} \frac{d^{n}\left(x^{2}-1\right)}{d x^{n}} \quad \forall x \in[-1,1]
$$

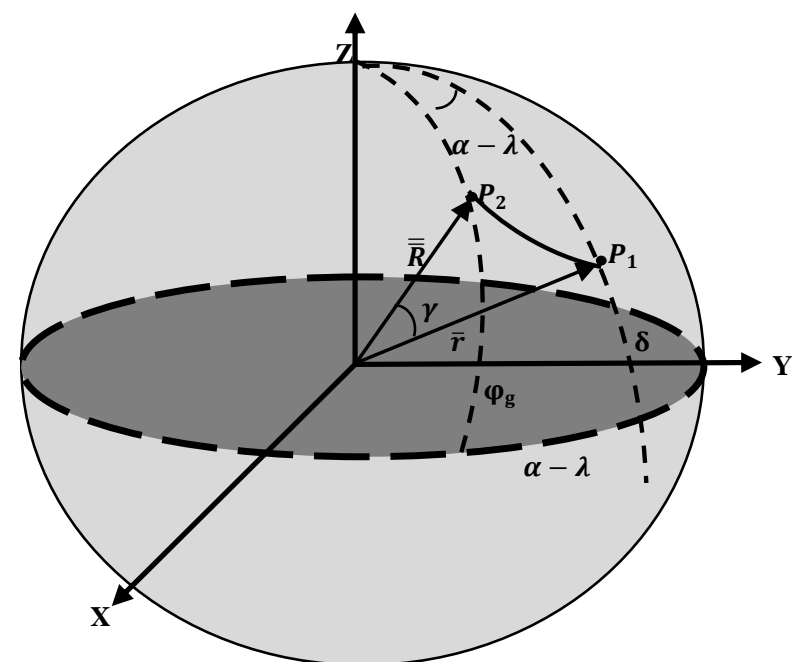

Fig. 2: Spherical Trigonometry of Satellite Position. $P_{1}$ Is the Satellite Position in Space While $P_{2}$ Is the Position of the Firing Station.

As illustrated in fig. 2, using the spherical triangle $\mathrm{P}_{1} \mathrm{P}_{2}$,

$\operatorname{Cos} \gamma=\operatorname{Sin} \varphi_{g} \operatorname{Sin} \delta+\operatorname{Cos} \varphi_{g} \operatorname{Cos} \delta \operatorname{Cos}(\alpha-\lambda)$

where $\boldsymbol{\alpha}$ and $\delta$ are the satellite's right ascension and declination of the satellite's position, while $\varphi_{\mathbf{g}}$ and $\boldsymbol{\lambda}$ is the ground station latitude and longitude. After simple mathematical treatments, we get:

$\mathrm{u}(\mathrm{r})=\frac{3 \varepsilon \Psi_{1}}{\mathrm{r}} \sum_{\mathrm{n}=0}^{\infty} \sum_{\mathrm{k}=0}^{\mathrm{n}}\left(\frac{\mathrm{R}}{\mathrm{r}}\right)^{\mathrm{n}} \mathrm{P}_{\mathrm{nk}}(\sin \delta)\left(\mathrm{C}_{\mathrm{nk}} \cos \mathrm{k} \alpha+\right.$ $\left.\mathrm{S}_{\mathrm{nk}} \sin \mathrm{k} \alpha\right)$

The coefficients $C_{n k}$ and $S_{n k}$ are dimensionless functions of latitude and longitude of the laser firing station and are defined as:

$C_{n k}=\frac{6}{\delta_{k}} \frac{(n-k) !}{(n+k) !} P_{n k}\left(\operatorname{Sin} \varphi_{s}\right) \operatorname{Cos} k \lambda$

$S_{n k}=\frac{6}{\delta_{k}} \frac{(n-k) !}{(n+k) !} P_{n k}\left(\operatorname{Sin} \varphi_{s}\right) \operatorname{Sin} k \lambda$ 
The parameter $\delta_{\mathrm{k}}$ has the values:

$\delta_{k}= \begin{cases}2 & \text { for } k=0 \\ 1 & \text { for } k>0\end{cases}$

For $\mathrm{n}=0$ and $\mathrm{k}=0$, after calculating $C_{00}, S_{00}$ and $P_{00}$, the potential

(9) can be developed to be:

$\mathrm{u}(\mathrm{r})=\frac{\varepsilon \Psi_{1}}{\mathrm{r}}[3+$

$\sum_{\mathrm{n}=1}^{\infty}\left(\frac{\mathrm{R}}{\mathrm{r}}\right)^{\mathrm{n}} \mathrm{C}_{\mathrm{n} 0} \mathrm{P}_{\mathrm{n}}(\sin \delta)+\sum_{\mathrm{n}=1}^{\infty} \sum_{\mathrm{k}=1}^{\mathrm{n}}\left(\frac{\mathrm{R}}{\mathrm{r}}\right)^{\mathrm{n}} \mathrm{P}_{\mathrm{nk}}(\sin \delta)\left(\mathrm{C}_{\mathrm{nk}} \cos \mathrm{k} \alpha+\right.$ $\mathrm{S}_{\mathrm{nk}} \sin \mathrm{k} \alpha$ )

As is clear from equations (10) and (11), the coefficients $C_{n k}$ and $S_{n k}$ are depending on the geocentric station coordinates, so we can call them the Station Harmonic Coefficients (SHC).

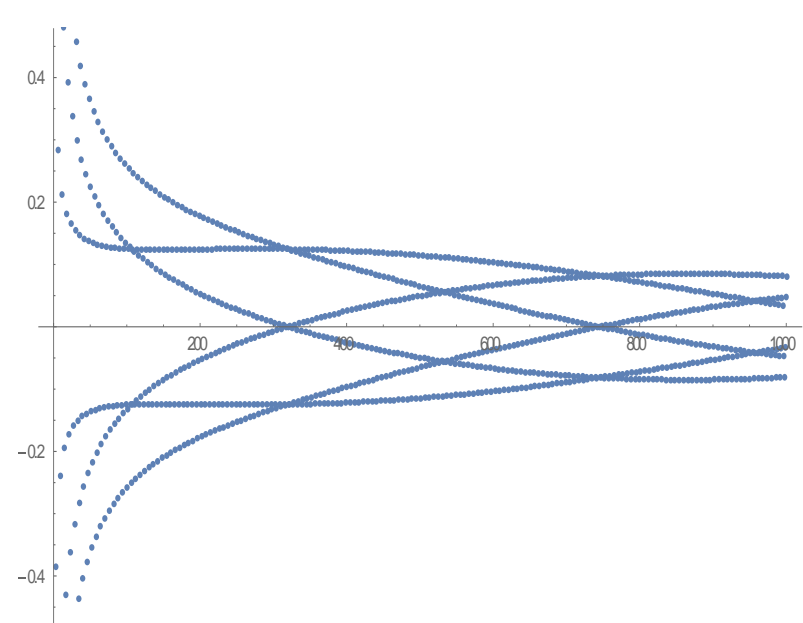

Fig. 3: The Variation of the Coefficients $C_{n o}$ with n.

For Helwan SLR station, the coefficients $C_{n 0}$, can be represented graphically as shown in Fig. 3.

Fig. 3 shows that the first term represents the maximum effects of the laser potential, so we will restrict our study on the zero and first $(n=1)$ terms of the potential $(13)$, then:

$\mathrm{u}(\mathrm{r})=\frac{3 \varepsilon \Psi_{1}}{\mathrm{r}}\left[1+\left(\frac{\mathrm{R}}{\mathrm{r}}\right) \mathrm{C}_{10} \mathrm{P}_{1}(\sin \delta)\right.$

$=\frac{3 \varepsilon \Psi_{1}}{\mathrm{r}}\left[1+\left(\frac{\mathrm{R}}{\mathrm{r}}\right) \sin \varphi_{\mathrm{g}} \sin \mathrm{I} \sin (\mathrm{f}+\omega)\right]$

With I, f, and $\omega$ are the inclination, true anomaly and the argument of perigee of the spacecraft's orbit respectively.

\section{The hamiltonian of the problem}

The Hamiltonian describing the motion of spacecraft under effects of the gravitational potential of spherical Earth and laser radiation pressure can be constructed as:

$\mathrm{H}=\frac{1}{2} \mathrm{P}^{2}+\mathrm{U}$

Where $\mathrm{P}$ is the canonical momentum and the total potential $\mathbf{U}$ is given by:

$\mathrm{U}=\frac{\mu}{\mathrm{r}}+\mathrm{u}(\mathrm{r})$

Where $\mu$ is the Earth's gravitational constant and $r$ is the magnitude of satellite position vector.

The Hamiltonian in terms of the Delaunay variables

$\left(\mathrm{l}_{1}, \mathrm{l}_{2}, \mathrm{l}_{3}, \mathrm{~L}_{1}, \mathrm{~L}_{2}, \mathrm{~L}_{3}\right)$, with $\left(\mathrm{l}_{1}, \mathrm{l}_{2}, \mathrm{l}_{3}\right)$ being the mean anomaly, $\boldsymbol{\omega}$ and $\Omega$ while $\left(\mathrm{L}_{1}, \mathrm{~L}_{2}, \mathrm{~L}_{3}\right)$ are their conjugate momentum respectively, expressed as:

$\mathrm{H}=\frac{\mu^{2}}{2 \mathrm{~L}_{1}^{2}}+\frac{3 \varepsilon \Psi_{1}}{\mathrm{r}}\left[1+\frac{\mathrm{S}}{\mathrm{r}} \Phi \sin \left(\mathrm{f}+\mathrm{l}_{2}\right)\right]$

With $\mathrm{S}=\operatorname{Sin} \mathrm{I}$ and $\Phi=R \operatorname{Sin} \varphi_{\mathrm{g}}$.

In order to apply the methods of canonical transformation based on Hor's technique, we need to study the orders of different terms of the Hamiltonian H. Let $\varepsilon$ be the small parameter of the problem defined as $\varepsilon=\frac{\mathrm{P}_{0}}{\pi \mathrm{C}}$. It is varied according to the laser space application as mentioned in the table (ftp://cddis.gsfc.nasa.gov/reports/slrlog/hlwl_20160727.log, ftp://cddis.gsfc.nasa.gov/reports/slrlog/oldlog/mdol_20040701.log and [6]):

Table 1: Small Parameter for Different Laser Applications

\begin{tabular}{|c|c|c|c|}
\hline $\begin{array}{l}\text { Laser space } \\
\text { applications }\end{array}$ & $\begin{array}{l}\text { Minimum } \\
\text { power }(\mathrm{KW})\end{array}$ & $\begin{array}{l}\text { Maximum } \\
\text { power }(\mathrm{KW})\end{array}$ & $\begin{array}{l}\text { Average small } \\
\text { parameter " } \varepsilon "\end{array}$ \\
\hline $\begin{array}{l}\text { Debris collision } \\
\text { avoidance }\end{array}$ & 5 & 10 & $8.0 \times 10^{-6}$ \\
\hline $\begin{array}{l}\text { Satellite laser } \\
\text { ranging }\end{array}$ & 0.0008 & 0.015 & $8.4 \times 10^{-9}$ \\
\hline
\end{tabular}

Based on using the small parameter, the Hamiltonian can be expressed in the following series:

$\mathcal{H}=\sum_{j \geq 0} \frac{\varepsilon^{j}}{j !} \mathcal{H}_{j}$

With,

$\mathcal{H}_{0}=\frac{\mu^{2}}{2 L_{l}^{2}}$

$\mathrm{H}_{1}=\frac{3 \Psi_{1} \mu}{\mathrm{L}_{1}^{2}}\left[\frac{\mathrm{a}}{\mathrm{r}}+\left(\frac{\mathrm{a}}{\mathrm{r}}\right)^{2} \frac{\mathrm{S} \Phi \mu}{\mathrm{L}_{1}^{2}} \sin \left(\mathrm{f}+\mathrm{l}_{2}\right)\right]$

It is clear from equations (17) that the Hamiltonian doesn't depend on $1_{3}=\Omega$ i.e. laser force cannot change the argument of ascending node. So, $\mathrm{L}_{3}$ can be considered as a constant of motion.

\section{Numerical application}

\subsection{Application on low earth orbit satellite}

The Experimental Geodetic Satellite (EGS) known as AJISAI is a spherical passive satellite of $2.15 \mathrm{~m}$ diameter and a mass of 685.2 $\mathrm{kg}$ with COSPAR ID 8606101 and NORAD ID: 16908. It was launched on Aug 13, 1986, and its operations still continuous for solid Earth studies; crustal movements and plate tectonics. It carries 318 mirrors and 120 laser retroreflector assemblies having 1436 corner cube reflectors. The mirror base made of an aluminum alloy and its surface has a protective coating of silicon oxide [11]. Its two-line elements is tabulated in table 2

Table 2: Ajisai Two Line Elements on 2016-11-21 (Http://Celestrak.Com). 16908 1986-061A AJISAI (EGS)

Launched: 1986-08-12 (224) Start Date: 2016-11-20 (325)

Decayed: Stop Date: 2016-11-21 (326)

1 16908U 86061A 16325.89358208 -.00000110 00000-0 -92752-4 09998

21690850.009395 .01290011192283 .8266186 .345712 .44484744

45508

1 16908U 86061A 16326.94929697-.00000100 00000-0 -16596-4 09992

21690850.009091 .76620011191286 .5504236 .063512 .44484692 45428

Laser potential and Hamiltonian have been estimated under the following simplifying assumptions:

a) A ground-based laser beam is fired towards the satellite as it was in the perigee. 
b) The absorbed light does not re-emitted again [12].

c) The optical properties of each elementary surface can be expressed by a linear combination of black body, a perfect mirror and Lambert diffuser [12].

d) The radiation assumed to fall normally to all satellite surfaces.

e) The metal mirror plates are perfectly reflecting(i.e. it has a reflectivity $\rho_{m} \sim 1$ ) with specularity $\beta_{m} \sim 1$.

f) The dimensions of the metal mirror plates are chosen to be that of the LRE satellite (http://god.tksc.jaxa.jp/lr/top.html).

g) The retro-reflector array is perfectly reflecting (i.e. it has a reflectivity $\rho_{r} \sim 1$ ) with specularity $\beta_{r} \sim 1$.

h) The reflecting area of each corner cube is $A_{c}=2 \sqrt{3} q^{2}$, where $q^{\prime}$ is the radius of the inscribed circle $\left(q^{\prime}=13.5 \mathrm{~mm}\right)$. (http://ilrs.gsfc.nasa.gov/docs/retromtg_060406_slides.pdf\#search $=\% 22$ ILRS $\% 20$ Meeting $\% 20$ on $\% 20$ Retroreflector\%20Arrays $\% 22$ ) In the present application, different sources of a ground-based laser are used; the laser system of the SLR stations of Helwan, Riyadh, Moblas-5, McDonald, Wettzell and Simosato. Laser system information of these stations are mentioned in table 3 (http://ilrs.gsfc.nasa.gov/network/stations/active/index.html).

Table 3: Laser System Information of Different SLR Stations

\begin{tabular}{|c|c|c|c|c|}
\hline \multirow[b]{2}{*}{$\begin{array}{l}\text { SLR Sta- } \\
\text { tion code }\end{array}$} & \multirow[b]{2}{*}{$\begin{array}{l}\text { Location and } \\
\text { Country }\end{array}$} & \multicolumn{2}{|c|}{ Laser system Information } & \multirow[b]{2}{*}{$\begin{array}{l}\text { Max Laser } \\
\text { power "W" }\end{array}$} \\
\hline & & $\begin{array}{l}\text { Max } \\
\text { energy } \\
\text { "mJ" }\end{array}$ & $\begin{array}{l}\text { Max repeti- } \\
\text { tion rate } \\
\text { "Hz" }\end{array}$ & \\
\hline HLWL & Helwan - Egypt & 80 & 5 & 0.4 \\
\hline RIYL & $\begin{array}{l}\text { Riyadh, Saudi } \\
\text { Arabia }\end{array}$ & 250 & 12 & 3 \\
\hline YARL & $\begin{array}{l}\text { Yarragadee, } \\
\text { Australia } \\
\text { McDonald }\end{array}$ & 200 & 10 & 2 \\
\hline MDOL & $\begin{array}{l}\text { Observatory, } \\
\text { Texas }\end{array}$ & 1500 & 10 & 15 \\
\hline WETL & $\begin{array}{l}\text { Wettzell, Ger- } \\
\text { many (WLRS) }\end{array}$ & 200 & 20 & 4 \\
\hline SISL & Simosato- Japan & 120 & 10 & 1.2 \\
\hline
\end{tabular}

Assuming that laser beam is fired toward the satellite as it was in its perigee, the Hamiltonian values are tabulated in table 4.

Table 4: Ground-Based Laser Potential on LEO Satellite Using Different SLR Station Laser Systems

\begin{tabular}{lc}
\hline SLR Station code & Laser potential "Joule" \\
\hline HLWL & $4 \times 10^{-14}$ \\
RIYL & $2.9 \times 10^{-13}$ \\
YARL & $1.1 \times 10^{-13}$ \\
MDOL & $1.5 . \times 10^{-12}$ \\
WETL & $4.5 \times 10^{-13}$ \\
SOSW & $1.2 \times 10^{-13}$ \\
\hline
\end{tabular}

As it clear in table 4, the laser potential increases as the laser energy and repetition rate increase. For single laser shot, Ajisai gains an average laser potential of about $4.18 \times 10^{-13} \cong \mathrm{O}\left(\mathrm{J}_{2}^{4}\right)$.

Using Mc Donald laser system, the laser potential on the satellite is illustrated in fig. 4 .

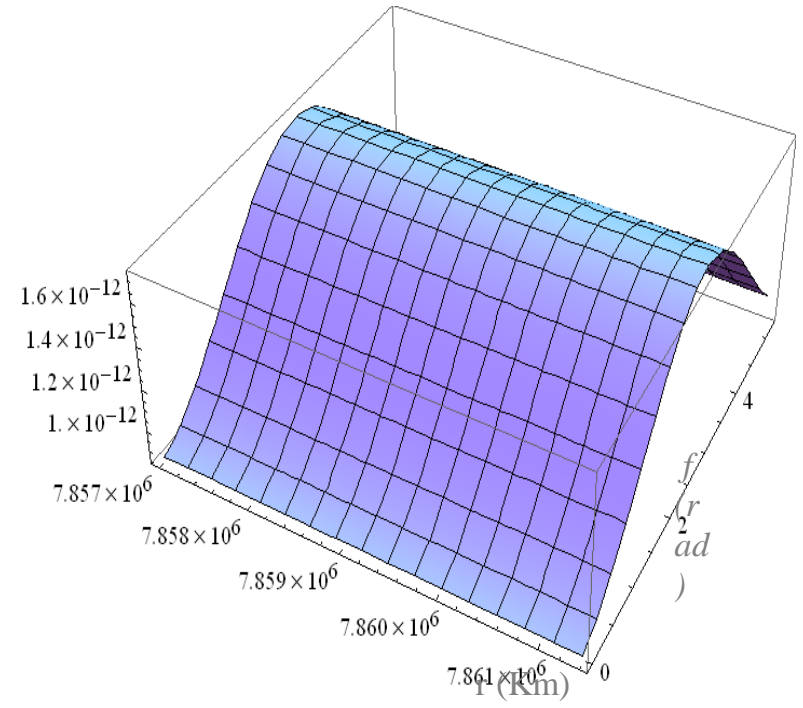

Fig. 4: ground-based laser potential over one revolution of LEO Satellite using the laser system of McDonald SLR station

Over one revolution, the satellite gains a maximum laser potential of about $1.7 \times 10^{-12} \cong \mathrm{O}\left(\mathrm{J}_{2}^{4}\right)$ as it is clear in fig. 4 .

\subsection{Application on LEO space Debris}

Assuming that a laser system is built on Helwan SLR station, with a laser power of about $5 \mathrm{~kW}$, in order to remove LEO space debris ( e.g. ASTRO-F DEB with NORD ID 29054). The accumulated laser potential on LEO debris is estimated over different intervals of time.

Table 5: Two Line Elements of ASTRO-F DEB with NORD ID 29054 on 2014-01-02 (Http://Celestrak.Com)

29054 2006-005E ASTRO-F DEB [LENS COVER]

Launched: 2006-02-21 (052) Start Date: 2014-01-02 (002)

Decayed: Stop Date: 2014-01-03 (003)

1 29054U 06005E 14002.16916607.00001553 00000-0 34531-3 02417

22905498.235610 .3600001052350 .2050310 .007414 .58342766417977

The accumulated laser potential over one revolution is about $10^{-9} \cong \mathrm{O}\left(\mathrm{J}_{2}^{3}\right)$ and after one day, it reaches about $10^{-8}$ as it is clear in table 6 .

Table 6: Accumulated Laser Potential on LEO Debris over Different Periods.

\begin{tabular}{ll}
\hline Interval & Laser potential "Joule" \\
\hline 1 revolution & $6.21 \times 10^{-10}$ \\
5 revolutions & $2.66 \times 10^{-9}$ \\
10 revolutions & $5.33 \times 10^{-9}$ \\
1 day & $7.81 \times 10^{-9}$ \\
\hline
\end{tabular}

\section{The averages effects}

To study the average effects of the laser radiation pressure we must take the average over one revolution of the satellite in its orbit. So, using the average technique, by averaging of equation (17) we get:

$\mathcal{H}^{*}=\sum_{j \geq 0} \frac{\varepsilon^{j}}{j !} \mathcal{H}_{j}^{*}$,

Where,

$\mathcal{H}_{j}^{*}=\frac{1}{2 \pi} \int_{0}^{2 \pi} \mathcal{H}_{j} d l_{1}$,

After calculating the required averages, we get the averaged Hamiltonian: 


$$
\mathcal{H}^{*}=\frac{\mu^{2}}{2 L_{l}^{2}}+\frac{3 \Psi_{1} \mu^{2}}{L_{l}^{4}}
$$

And the system of Hamilton's equation of motion will be:

$$
l_{i}=\frac{\partial \mathcal{H}^{*}}{\partial L_{i}} \quad \& \quad L_{i}=-\frac{\partial \mathcal{H}^{*}}{\partial l_{i}}, \quad i=1,2,3
$$

From the second group of eqs. (20), the laser will not affect the momenta of the orbital elements, while it will affect the mean anomaly, $1_{1}$, only. The rate of change in the mean anomaly due to laser pressure is:

$$
i_{1}=-\frac{\mu^{2}}{L_{I}^{3}}-\frac{12 \Psi_{1} \mu^{2}}{L_{I}^{5}}
$$

The last result needs a numerical check and higher order of the laser pressure need to study because we expect to found changes in the other elements in higher orders.

\section{Discussion and conclusion}

In this study, the ground-based laser force is proved to be a conservative force in case of single laser shot. Consequently, the associated Hamiltonian is formulated.

The implementation of the model for a single laser shot shows that:

1) For SLR systems:

- As the repetition rate of the laser system increase, laser force is increasing as well.

- For single laser shot, the average potential has approximately same order as $\mathrm{J}_{2}^{4}$.

- Over one satellite revolution, the average potential has approximately same order as $\mathrm{J}_{2}^{4}$.

2) For space debris removal

- As the explosure time increase, laser potential can be accumulated to be of a significant value.

- The estimated value of laser potential per day has approximately same order as $\mathrm{J}_{2}^{3}$.

In general, some adaptations and development of laser systems such as their energies, repetition rates,..., etc are necessary in order to perform the required perturbations of the satellite or debris orbital dynamics which enable space debris removal.

\section{References}

[1] Jonathan W. Campbell (2000) Using Lasers in Space: Laser Orbital Debris Removal and Asteroid Deflection. Occasional Paper No. 20 Center for Strategy and Technology Air War College.

[2] El-Saftawy M.I., and Makram Ibrahim (2004) "The laser shots as a perturbing force on spacecraft's orbit", NRIAG J. of Astronomy and Astrophysics, Special issue.

[3] El-Saftawy M.I. (2006) Orbital perturbation due to laser pressure in absence of Earth's atmosphere ", NRIAG J. of Astronomy and Astrophysics, special issue.

[4] El-Saftawy M.I. and Nabawia S. Khalif (2008) Artificial Radiation Effect on Spacecraft's Orbit considering atmospheric beam attenuation. First Middle East and Africa IAU-Regional Meeting Proceedings MEARIM No. 1.

[5] Nabawia S. khalifa (2009) Effect of an Artificial Radiant Force on the Spacecraft's Orbit. PhD. Thesis, Cairo University.

[6] James Mason, Jan Stupl, William Marshall and Creon Levit (2011) Orbital Debris-Debris Collision Avoidance. Journal of Advances in Space Research, vol. 48. https://doi.org/10.1016/j.asr.2011.08.005.

[7] El-Saftawy M. I., Afaf M. Abd El-Hameed, and Nabawia S. Khalifa (2007) Analytical Studies of Laser Beam Propagation through the Atmosphere. Proceeding of 6th International Conference on Laser Science and Applications (ICLAS-07), Cairo, Egypt.

[8] Danielson E., Levin J., Abrams E. (2003) Meteorology second edition, McGraw-Hill Companies.

[9] Prilutsky O.F. and Fomenkova M.N. (1990) Appendix laser beam scattering in the atmosphere. Science \& Global Security, Vol. 2. https://doi.org/10.1080/08929889008426349.

[10] Escobal P.R. (1965) Methods of Orbit Determination. John Wiley and Sons, Inc., New York, London, Sydney.

[11] Sasaki, M and Hashimoto H. (1987) Launch and Observation of the Experimental Geodetic Satellite of Japan. IEEE Transactions on Geoscience and Remote Sensing, Volume 25, No. 5. https://doi.org/10.1109/TGRS.1987.289830.

[12] Milani, A., Nobili, A.M. and Farinella, P. (1987) non-gravitational perturbations and satellite geodesy, IOP Publishing Ltd. 